\title{
Interactive comment on "Methane emissions from an oil sands tailings pond: A quantitative comparison of fluxes derived by different methods" by Yuan You et al.
}

Yuan You et al.

ralf.staebler@canada.ca

Received and published: 20 October 2020

Please see the pdf below for responses

Please also note the supplement to this comment:

https://amt.copernicus.org/preprints/amt-2020-116/amt-2020-116-AC1-

supplement.pdf 